Journal for ImmunoTherapy of Cancer

\title{
HER2-LAMP vaccines effectively traffic to endolysosomal compartments and generate enhanced polyfunctional $T$ cell responses that induce complete tumor regression
}

\author{
Alan Chen Chen, ${ }^{1}$ Renhuan Xu, ${ }^{2}$ Tao Wang, ${ }^{1}$ Junping Wei, ${ }^{1}$ Xiao-Yi Yang, ${ }^{1}$ \\ Cong-Xiao Liu, ${ }^{1}$ Gangjun Lei, ${ }^{1}$ Herbert Kim Lyerly, ${ }^{1,3,4}$ Teri Heiland, ${ }^{2}$ \\ Zachary Conrad Hartman (i) ${ }^{1,3}$
}

To cite: Chen AC, Xu R, Wang T, et al. HER2-LAMP vaccines effectively traffic to endolysosomal compartments and generate enhanced polyfunctional $\mathrm{T}$ cell responses that induce complete tumor regression. Journal for ImmunoTherapy of Cancer 2020;8:e000258. doi:10.1136/ jitc-2019-000258

- Additional material is published online only. To view please visit the journal online (http://dx.doi.org/10.1136/jitc2019-000258).

ACC and $\mathrm{RX}$ are joint first authors.

Accepted 01 May 2020

Check for updates

(C) Author(s) (or their employer(s)) 2020. Re-use permitted under CC BY-NC. No commercial re-use. See rights and permissions. Published by BMJ.

For numbered affiliations see end of article.

\section{Correspondence to}

Dr Zachary Conrad Hartman, Hartman MSRBI Room 414 Box 2606, Durham, North Carolina 27710, USA;

zachary.hartman@duke.edu

\section{ABSTRACT}

Background The advent of immune checkpoint blockade antibodies has demonstrated that effective mobilization of $T$ cell responses can cause tumor regression of metastatic cancers, although these responses are heterogeneous and restricted to certain histologic types of cancer. To enhance these responses, there has been renewed emphasis in developing effective cancer-specific vaccines to stimulate and direct $\mathrm{T}$ cell immunity to important oncologic targets, such as the oncogene human epidermal growth factor receptor 2 (HER2), expressed in $\sim 20 \%$ of breast cancers (BCs).

Methods In our study, we explored the use of alternative antigen trafficking through use of a lysosome-associated membrane protein 1 (LAMP) domain to enhance vaccine efficacy against HER2 and other model antigens in both in vitro and in vivo studies.

Results We found that inclusion of this domain in plasmid vaccines effectively trafficked antigens to endolysosomal compartments, resulting in enhanced major histocompatibility complex (MHC) class I and II presentation. Additionally, this augmented the expansion/ activation of antigen-specific CD4+ and CD8+ T cells and also led to elevated levels of antigen-specific polyfunctional CD8+ T cells. Significantly, vaccination with HER2-LAMP produced tumor regression in 30\% of vaccinated mice with established tumors in an endogenous model of metastatic HER2+ BC, compared with $0 \%$ of HER2-WT vaccinated mice. This therapeutic benefit is associated with enhanced tumor infiltration of activated CD4+ and CD8+ T cells.

Conclusions These data demonstrate the potential of using LAMP-based endolysosomal trafficking as a means to augment the generation of polyfunctional, antigenspecific T cells in order to improve antitumor therapeutic responses using cancer antigen vaccines.

\section{INTRODUCTION}

Approximately $\sim 20 \%$ of all breast cancers (BCs) are characterized by overexpression of the oncogene human epidermal growth factor receptor 2 (HER2), which plays a critical role in the transformation and maintenance of these cancers. ${ }^{1-3}$ Advanced HER2+ BCscan be treated with a combination of chemotherapy and HER2-specific monoclonal antibodies (mAbs), but these are not curative treatments for metastatic HER2+ BC. ${ }^{12}$ Additional HER2 targeted treatments (such as small molecule inhibitors and the HER2-Antibody Drug Conjugate, T-DM1) also have efficacy in metastatic HER2+ BC but are not curative, thus more effective therapies for HER2+ BC are needed. ${ }^{14}$ Although antigen loss or mutation are known mechanisms for therapeutic resistance, oncogenic HER2 expression remains elevated in therapy resistant cancers and is critical for their continued growth, making HER2 a compelling immunologic target. ${ }^{125}$ In our previous studies, we developed HER2-specific viral vaccines encoding inactivated and truncated forms of HER $2^{67}$ which we used to stimulate HER2-specific immune responses in patients with advanced HER2+ BC. ${ }^{6}$ These studies demonstrated that viral vaccines encoding truncated forms of HER2 could break HER2specific tolerance in HER2+ BC patients, with the induction of significant HER2-specific T cell memory responses in a subset of patients. Notably, we found that these HER2-specific memory responses were associated with enhanced progression-free survival. ${ }^{6}$ As the HER2 specific immune responses were only observed in a subset of patients, we wanted to explore strategies to enhance immune responses to HER2, and considered genetic modifications to our vaccine.

One genetic strategy to enhance vaccineinduced, antigen-specific $\mathrm{T}$ cell responses 
is by altering antigen trafficking to different subcellular compartments. ${ }^{8-10}$ Lysosome-associated membrane protein 1 (LAMP1) is a type 1 membrane protein possessing a short cytosolic $\mathrm{C}$-terminus containing an endolysosomal targeting sequence. LAMP antigen targeting is a means to direct expressed antigens containing LAMP trafficking signals into the endolysosomal compartments and enhance MHC class II pathway presentation. ${ }^{11} 12$ LAMP antigen trafficking circumvents the limitation of all nonexported proteins from viral and plasmid vaccines being routed to MHC class I pathways and enhances MHC class II presentation and subsequent stimulation of CD4+ T cell and B cell responses. ${ }^{11}{ }^{13}$ As MHC class II presentation has been linked to effective antitumor immunity ${ }^{14}$ and CD4+ Tcell responses associate with HER2-specific antitumor responses, ${ }^{15-18}$ LAMP-based vaccines have strong potential for use against HER2 and other cancer antigens. However, LAMP-based vaccines have been largely been used for pathogen targets, including HIV, HPV, cytomegalovirus (CMV), West Nile Virus, SARS, Hantavirus and Dengue virus. ${ }^{11} 12$ 19-24 These studies have collectively demonstrated an enhanced ability for LAMP vaccines to present antigens through the MHC class II pathway, resulting in improved pathogen-specific immune responses. More recent studies have used LAMP-based vaccines to skew immune responses to specific allergens, such as the Crly2 protein from Japanese Red Cedar pollen, toward a non-allergic Th1 response as a means to suppress $\operatorname{IgE}$ production. ${ }^{25}{ }^{26}$ Collectively, these studies suggest that integration of LAMP targeting into HER2 vaccines would enhance the induction of HER2-specific Th1 CD4 T cell responses, which may translate into more effective antitumor immunity. However, it was unclear if this strategy may be hampered by potentially altered or suppressed presentation through the MHC class I pathway, or if it would be sufficiently effective against non-pathogen antigens (that are subject to immune tolerance) to induce antitumor immunity.

In addition to enhancing MHC class II presentation, an additional benefit of antigen targeting is the ability to be incorporated into multiple vector platforms that have established production and safety profiles without the need to evaluate different experimental adjuvants or novel vector platforms. In the past, we explored the use of exosomal antigen trafficking and found that while it enhanced $\mathrm{T}$ cell and B cell responses to certain antigens, such as Carcinoembryonic Antigen, it did not strongly enhance responses compared with full length or truncated forms of HER2. ${ }^{8}$ Due to our clinical success in HER2 vaccination, ${ }^{6}$ we explored the endolysosomal trafficking of a HER2 vaccine through the incorporation of a LAMP trafficking motifs into a HER2 construct containing both the extracellular and intracellular domains (ECD/ICD) of the receptor. Our studies found that HER2-LAMP enhanced endolysosomal trafficking of HER2 compared with HER2-WT, which resulted in significantly decreased HER2 expression and enhanced both MHC class I and II presentation of LAMP-fusion peptides. This modification permitted greater early expansion of both antigenspecific CD4+ and CD8+ T cells in draining lymph nodes, which translated into greater systemic immune responses, as demonstrated by superior induction of polyfunctional HER2-specific $\mathrm{T}$ cells after HER2-LAMP vaccination. The enhanced quantity and quality of HER2-specific T cell responses after HER2-LAMP vaccination allowed for more effective antitumor immunity, dependent on both CD4+ and CD8+ T cells. Critically, HER2-LAMP vaccines produced significantly augmented antitumor responses against HER2 self-antigens, resulting in complete tumor regression in an aggressive endogenous model of HER2+ $\mathrm{BC}$ in a significant fraction of mice $(\sim 30 \%)$. This regression was associated with greater $\mathrm{CD} 4+$ and CD8+ Tcell activation within tumors of HER2-LAMP-vaccinated mice compared with tumors from HER2-WT-vaccinated mice. Thus, our study demonstrates that LAMP antigen trafficking can enhance $\mathrm{T}$ cell responses, producing expanded, multipotent HER2-specific T cells that enable effective antitumor immunity in a model of advanced HER2+ BC.

\section{MATERIALS AND METHODS Mice}

Female BALB/c, C57BL/6, OTI, OTII, MHCI-/-, MHCII-/- and HLA2.1 were purchased from Jackson Labs and bred at Duke in accordance with the Duke Institutional Animal Care and Use Committee (IACUC). The endogenous model of HER2+ BC was a kind gift from William Muller (McGill University). ${ }^{27}$ All study protocols involving mice were performed in accordance with IACUC guidelines.

\section{Vector construction}

DNA vaccines encoding HER2+LAMP and chicken ovalbumin (OVA)+LAMP were generated at Nature Technology Corporation (NTC, Lincoln, Nebraska, USA). The HER2 gene was codon optimized for human usage using the GeneArt/Invitrogen online gene design software. The synthetic genes were manufactured by GeneArt/ Invitrogen (Life Technologies, Grand Island, New York, USA). The HER2/LAMP construct was made by inserting the HER2 sequence between the luminal domain (Xho1) and the transmembrane/cytoplasmic domain (EcoR1) of LAMP in the expression vector NTC8382-VA1. NTC8382-VA1 is a covalently closed circular doublestranded plasmid vector. The flanking regions of the insertion site are the eukaryotic promoter (CMV) and poly-A transcriptional terminator that flank the insertion site to express LAMP fusion proteins in target cells. The plasmid was transformed into NTC4862 host cell line competent cells and selected for sucrose resistance.

\section{Plasmid vaccinations}

Intramuscular vaccinations requiring a single dose were performed using the BTX ECM 830 electroporation system while intramuscular vaccinations requiring 
multiple doses were performed using the ICHOR TriGrid Delivery System adapted for vaccine delivery in mice. The right thigh was shaved, sterilized with $70 \%$ ethanol and injected with the vaccine followed 5 sec later by electroporation using the proprietary ICHOR electrical pulse settings. In the case of multiple boosts, the same electroporation procedure was performed on alternating thighs that is, right thigh on day 0 , left thigh on day 7 and right thigh on day 14. Immunological tests (Fluorescenceactivated cell sorting (FACS) analysis, interferon- $\gamma$ (IFN $\gamma$ ) ELISPOT, HER2-specific cell-based ELISA) were performed 7 days after the last boost of every experiment.

Intradermal vaccinations were performed by injection of DNA in a volume of $20 \mu \mathrm{L}$ into the ventral surface of the ear followed by electroporation at the site of injection using ICHOR system. Immunological tests were performed 7 days after the last boost of every experiment.

\section{Orthotopic transplantation and antibody depletion}

$\mathrm{BALB} / \mathrm{c}$ mice were injected with TSA cells (american type culture collection (ATCC)), which were stably transduced with lentivirus expressing a wild-type human ErBB2 gene. Antimouse CD4 (clone GK1.5), antimouse CD8 (clone 2.43) and isotype control IgG were purchased from Bio X Cell. Mice were depleted of $\mathrm{T}$ cell subsets in vivo by intraperitoneal injection with $250 \mu \mathrm{g}$ of anti-CD4 (GK1.5) or anti-CD8 (2.43) mAbs on days -3, 1, 5, 8 and twice a week after tumor injection. An implantation success rate of $100 \%$ was observed for all models in all treatment groups with the exception of 1 HER2-LAMP vaccinated mouse in the induction model.

\section{Mouse IFN $\gamma$ ELISPOT assay}

Mouse splenocytes (500,000-1,000,000 cells/well) were isolated and stimulated in RPMI1640 medium with 10\% FBS for 24-48 hours with the following peptides: HER2 ICD pooled peptides $(1 \mu \mathrm{g} / \mathrm{mL}$; JPT), HER2 ECD pooled peptides $(1 \mu \mathrm{g} / \mathrm{mL}$; JPT), irrelevant HIV-gag peptide mix $(1 \mu \mathrm{g} / \mathrm{mL}: \mathrm{JPT})$ or PMA $(50 \mathrm{ng} / \mathrm{mL})$ and ionomycin $(1 \mu \mathrm{g} / \mathrm{mL}$; Sigma) as positive controls. The HER2 ECD and ICD peptide used in the ELISPOT assay were in a 1:1 proportion. The mouse IFN $\gamma$ ELISPOT assay kit (Mabtech) was used.

\section{Cell-based ELISA of anti-HER2 antibodies}

Serum from vaccinated mice was diluted and added to parental or human HER2-expressing NMUMG cells for 1 hour at room temperature. Plates were washed and cells were fixed with $1 \%$ formalin. A secondary antimouse IgGHRP conjugated antibody (Cell Signaling Technology) was used prior to developing with 3,3',5,5'-Tetramethylbenzidine (TMB) substrate (Biolegend) and absorbance determined using a Bio-Rad Model 680 microplate reader (Bio-Rad).

\section{Immunofluorescence microscopy}

293T, E0771, and JAWSII cells were grown on 8-well slides (MatTek) coated with $1 \mu \mathrm{g} / \mathrm{mL}$ human fibronectin (EMD Millipore). Transfection was performed using
Lipofectamine3000 (Thermofisher). For Lysotracker (ThermoFisher) staining, cells were incubated with $75 \mathrm{~nm}$ Lysotracker for 1 hour at $37^{\circ} \mathrm{C}$. For endoplasmic reticulum (ER) staining, cells were incubated with $2 \mu \mathrm{L}$ ER CellLight ER-RFP Bacman V.2.0 (Thermofisher) for 24 hours. Slides were washed with phosphate buffered saline (PBS), fixed with $10 \%$ formalin for $20 \mathrm{~min}$ and mounted with DAPI Fluoromount-G (SouthernBiotech). Staining was performed in $0.1 \%$ PBST with rabbit anti-HER2 (1:200, Cell Signaling), rat anti-LAMP1 (1:200, Abcam) or rat anti-MHCII (1:200, Thermofisher) at $4^{\circ}$ overnight and antirabbit Alexa Fluor 488 (1:1000 dilution, Invitrogen) or antirat Texas Red-X (1:1000, invitrogen) at room temperature for 1 hour. Slides were mounted with Fluoromount-G with DAPI (Southern Biotech).

\section{Western blot}

Tissue culture cells were harvested and lysed using RIPA buffer. Total lysate $(20 \mu \mathrm{g})$ was ran on a gel using the Biorad Mini-PROTEAN system, followed by transfer using the Biorad semi dry transfer system. Primary antibodies used were rabbit anti-HER2 (1:1000, Cell Signaling), mouse anti-OVA (1:1000, Abcam) or rabbit anti-GAPDH (1:1000, Cell Signaling). Antirabbit IRDye 800 (1:1000, LICOR) and antimouse IRDye 680 (1:1000, LICOR) were used as secondaries. Blocking, probing, visualization and quantification were performed according to LI-COR Odyssey system specifications.

\section{Flow cytometry}

For flow cytometry of tissue culture cells, cells were isolated from mouse spleens and mechanically dissociated with a $40 \mu \mathrm{m}$ cell strainer (Greiner Bio-One). Red blood cells were lysed with RBC lysing buffer (Sigma). Cells were labeled with aqua live/dead fixable viability dye in PBS (1:500), followed by surface antibodies (1:100) in staining buffer (1\% BSA, $1 \%$ rat serum in PBS). Antibodies used for surface staining: PE/Dazzle anti-CD4 (RM4-5, Biolegend), APC/CY7 anti-CD8 (YTS156.7.7, Biolegend), PE/CY7 anti-CD44 (IM7, Biolegend), antiCD69 (H1.2F3, BD Bioscience). For intracellular staining, cells were stained with aqua live/dead dye, followed by surface staining, fixation with perm/fixation solution (Thermofisher), and stained with intracellular antibodies against FITC anti-IFN $\gamma$ (XMG1.2, Invitrogen) and AF647 anti-tumor necrosis factor $\alpha(\mathrm{TNF} \alpha)$ (MP6-XT22, Biolegend) in $1 \mathrm{x}$ perm/wash buffer. Cells were washed and analyzed on a CytoFLEX flow cytometer (Beckman Coulter) and analyzed using FlowJo software.

\section{Statistical analysis}

Statistical analyzes for all experiments were performed with the indicated statistical tests using Graphpad Prism software. Data are means \pm SEM tumor volumes, flow cytometry, ELISA and ELISPOT data from experiments with three or more treatment groups were analyzed by one-way analysis of variance with Bonferroni's multiple comparisons test. A two-tailed, unpaired Student's 


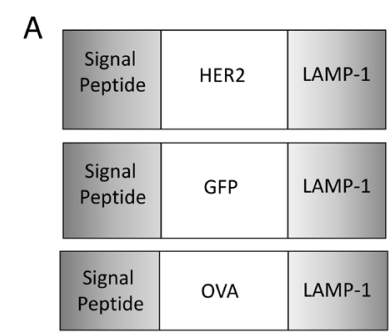

B
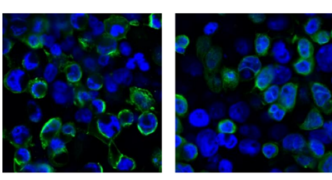
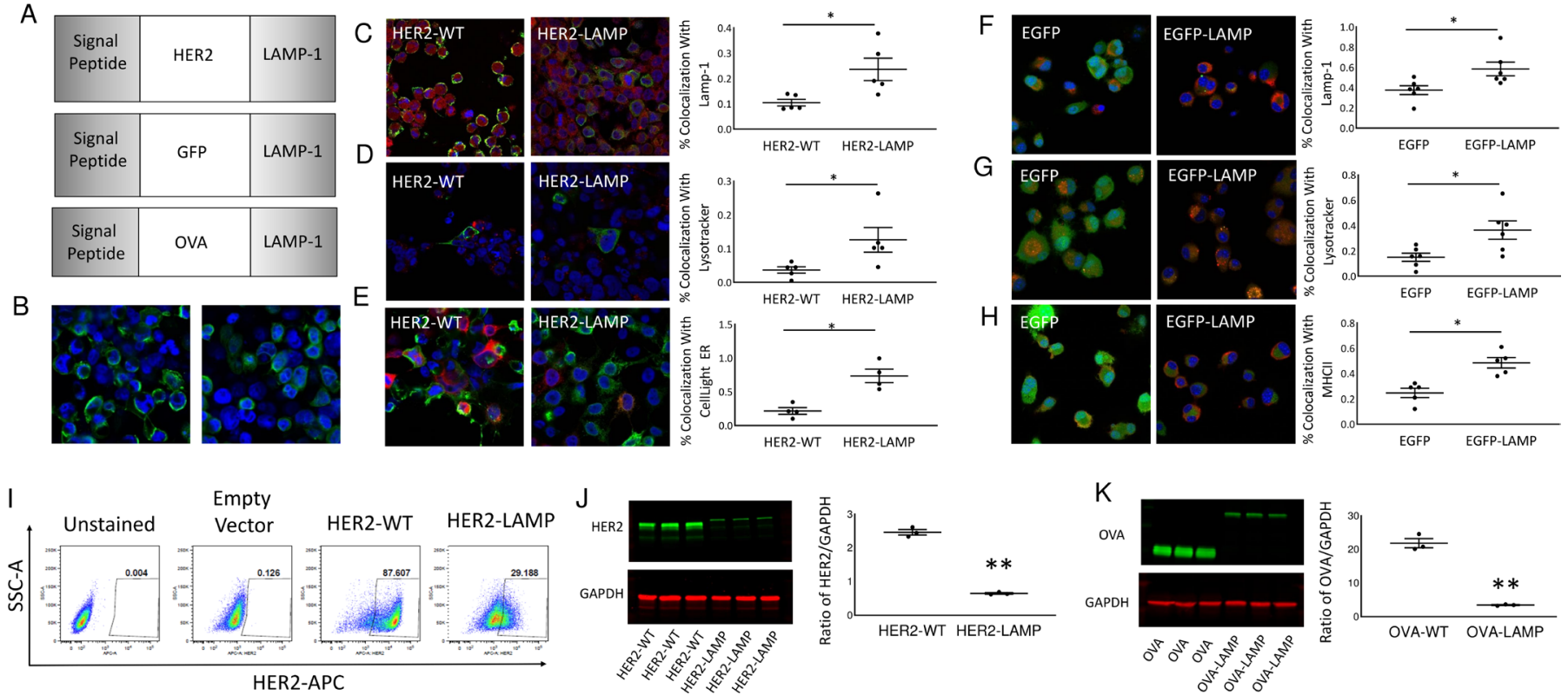

HER2-APC

Figure 1 Altered antigen trafficking and expression via LAMP. (A) Diagram representations of HER2-LAMP, OTI/OTII-GFPLAMP and OVA-LAMP DNA plasmids. (B) Subcellular distribution of HER2 in HER2-WT or HER2-LAMP transfected 293T cells as detected by immunofluorescence microscopy, $n=3$ (C) Colocalization of HER2 with endogenous LAMP-1 $n=5$, Lysotracker (D) $n=5$ and CellLight ER (E) $n=4$ markers in 293T cells transfected with HER2-WT or HER2-LAMP, along with their respective quantification of colocalization normalized by total expression of HER2. (F) Colocalization of GFP with endogenous LAMP-1 $n=6$, Lysotracker $(G) n=6$ and MHCII $(H) n=5$ markers in JAWSII cells stably transduced with OTI/OTII-EGFP or OTI/OTII-EGFPLAMP, along with their respective quantification of co-localization normalized by total expression of EGFP. (I) Flow cytometric analysis of surface HER2 expression of 293T cells transfected with HER2-WT or HER2-LAMP, $n=3$. (J) Western blot of HER2 expression of 293T cells transfected with HER2-WT or HER2-LAMP with their respective quantification, $n=3(K)$ Western blot of OVA expression of 293T cells transfected with OVA-WT or OVA-LAMP with their respective quantification, $n=3$. ER, endoplasmic reticulum; HER2, human epidermal growth factor receptor 2; LAMP, lysosome-associated membrane; OVA, ovalbumin. Student t-test. ${ }^{*} p<0.05,{ }^{* *} p<0.01$.

t-test, was used for experiments with only two groups. Tumor volumes were analyzed at the terminal endpoint only, unless otherwise indicated. Statistical analysis was performed using Prism (GraphPad). P values of 0.05 or less were considered statistically significant. *, $\mathrm{p}<0.05$; **, $\mathrm{p}<0.01 ; * * *, \mathrm{p}<0.001$. Statistical tests: two-tailed Student t-test. $* \mathrm{p}<0.05, * * \mathrm{p}<0.01$.

\section{RESULTS \\ Generation of lamp vectors with altered antigen trafficking and expression}

As previous studies have demonstrated that LAMP1 trafficked antigens can alter responses to infectious disease and allergy, ${ }_{11} 1323242628-30$ we wanted to determine if this strategy could alter immune responses to credentialed endogenous tumor antigens, such as HER2. To first test altered trafficking, we generated a series of LAMPtargeted and control constructs by adding an LAMP signaling sequence to the N-terminus and the LAMP ICD to the C-terminus (figure 1A). These genes were cloned into CMV-promoter expression plasmids and transfected to assess subcellular localization and trafficking by immunofluorescence microscopy. In these experiments, we noted an altered localization of HER2-LAMP in transfected $293 \mathrm{~T}$ cells, illustrated by a more intracellular distribution in contrast to the membrane-enriched distribution in native HER2-WT transfected cells (figure 1B). Colocalization studies revealed that HER2-LAMP significantly colocalized with native LAMP1 (figure 1C), lysosomes (figure 1D) and the ER (figure 1E), in comparison to HER2-WT. To determine if this altered trafficking depended on the transgene or cell type, we transfected OTI/II-GFP-LAMP and GFP into the dendritic cell (DC) line JAWSII. Colocalization studies of these cells again revealed that OTI/II-GFP-LAMP colocalized with native LAMP1 (figure 1F), lysosomes (figure 1G) and MHC class II (figure 1H) in comparison to an OTI/II-GFP control. To confirm altered cell surface trafficking of HER2, we also performed flow cytometry on 293T and RAW264.7 (macrophage) cells transfected with HER2-WT or HER2LAMP (Figure 1I, online supplementary S1A). We found that in both types of cells, HER2-LAMP had reduced cell surface expression compared with HER2-WT. In our microscopy and flow-based experiments, we also observed that LAMP vectors had reduced overall antigen expression intensity compared with control counterparts. To test if this altered trafficking led to reduced protein expression, we performed Western blot analysis on HER2 and HER2-LAMP transfected cells, as well as OVA-WT and OVA-LAMP transfected cells (figure $1 \mathrm{~J}-\mathrm{K}$ ). We found that 
although we could detect both proteins by Western blot, LAMP-targeted genes had significantly reduced levels of protein expression. We subsequently confirmed this by flow cytometry in GFP-WT and GFP-LAMP vectors, as well as by luciferase activity readings in cells transfected with luciferase-wild-type (LUC-WT) and LUC-LAMP vectors (online supplementary fig S1B-C). For every antigen and detection strategy, we observed significantly reduced expression of LAMP variants, suggesting that altered cellular trafficking to lysosomes leads to differences in expression that might contribute to antigen presentation and lead to altered antigen-specific immunity.
Vaccination with antigen-directed lamp vectors elicits significantly enhanced antigen-specific $\mathrm{T}$ cell responses, but weaker B cell responses in vivo

To determine if LAMP targeting alters $\mathrm{T}$ cell-specific immunity to tumor antigens by vaccination in a clinically relevant setting, we first investigated immune responses in C57BL/6-hHLA-2.1 mice, which express a human HLA-2.1 molecule for antigen presentation. We vaccinated these mice using intramuscular electroporation with HER2-WT or HER2-LAMP plasmids and assessed HER2specific immune responses (figure 2A). Splenocytes from

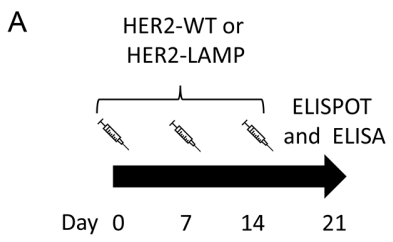

D

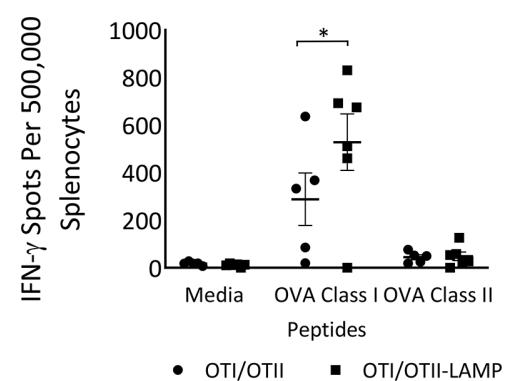

G

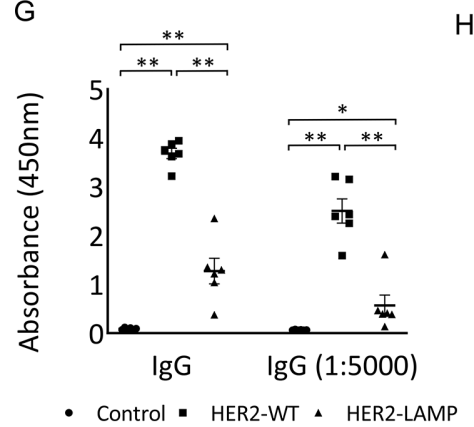

B

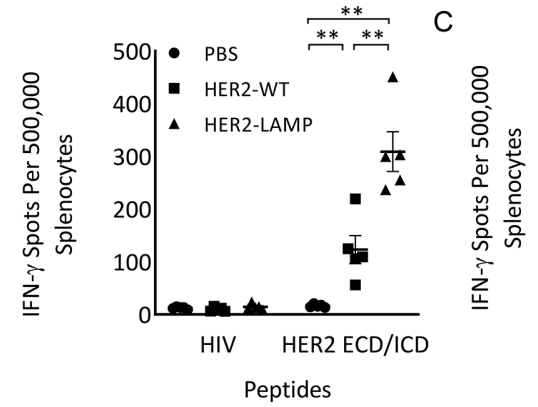

E

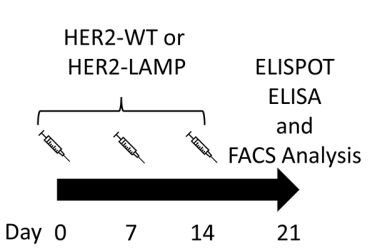

F
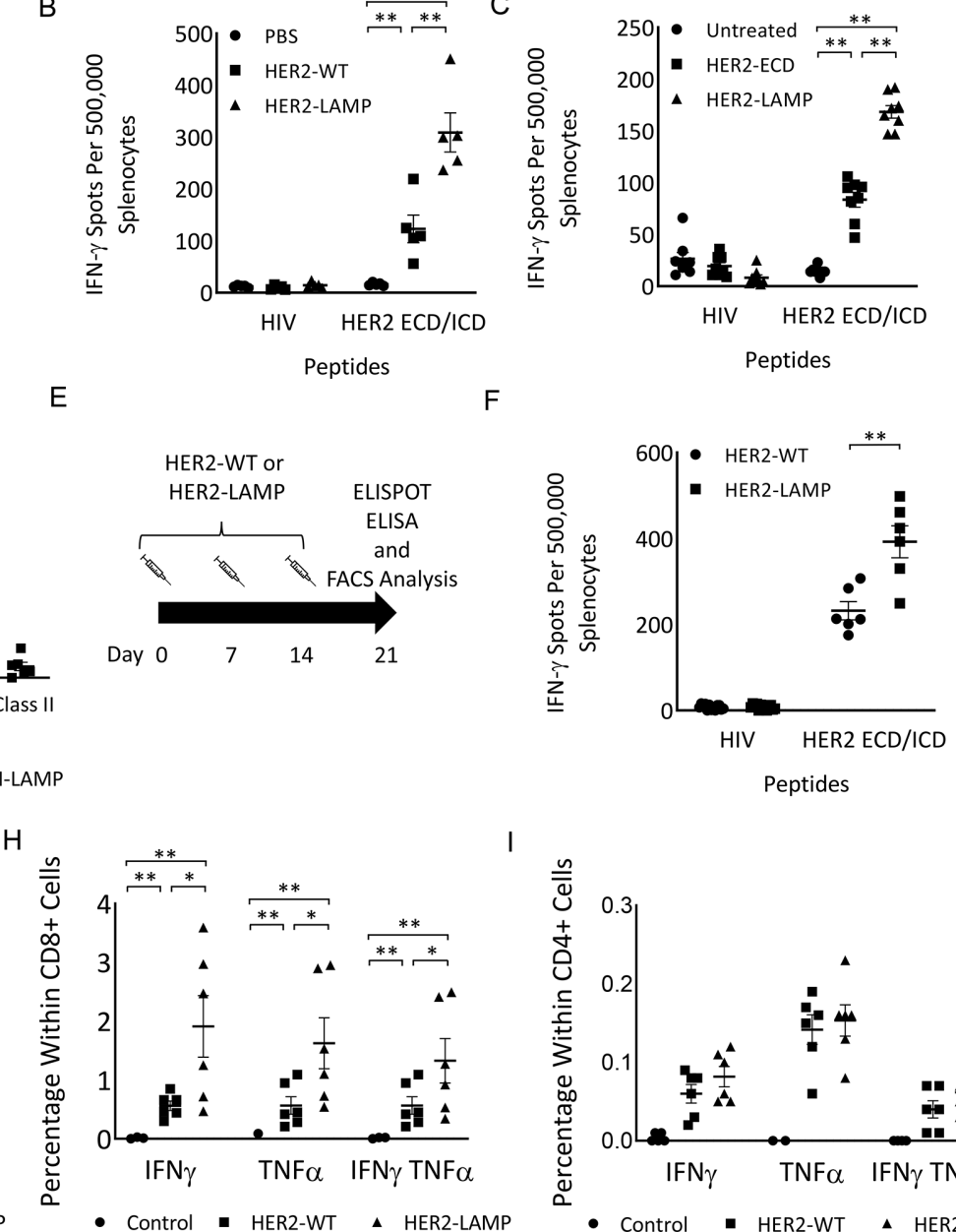

Figure 2 Enhanced T-cell and B-cell mediated immune responses elicited by HER2-LAMP vaccination. (A) HLA2.1 mice were vaccinated via intramuscular electroporation with PBS control, $40 \mu \mathrm{g}$ HER2-WT, or $40 \mu \mathrm{g}$ HER2-LAMP with 2 homologous boosts administered at 7 and 14 days after the initial vaccination. ELISPOT and ELISA were performed 7 days after the last vaccination. (B) ELISPOT detecting HER2-specific IFN $\gamma$ response in splenocytes of vaccinated HLA2.1 mice, $n=5$ (C) ELISPOT detecting HER2-specific IFN $\gamma$ response in splenocytes of C57BL/6 mice vaccinated with $50 \mu \mathrm{g}$ of HER2-ECD or HER2-LAMP, $\mathrm{n}=8$ (D) ELISPOT detecting antigen-specific IFN $\gamma$ response in splenocytes stimulated with class I or class II restricted OVA peptides from OVA-LAMP vaccinated C57BL/6 mice, $n=5$ (E) BALB/c mice were vaccinated via intradermal electroporation with $40 \mu \mathrm{g}$ control vector, $40 \mu \mathrm{g} \mathrm{HER2-WT}$, or $40 \mu \mathrm{g} \mathrm{HER2-LAMP} \mathrm{with} 2$ homologous boosts administered at 7 and 14 days after the initial vaccination. ELISPOT and ELISA were performed 7 days after the last vaccination. (F) ELISPOT detecting HER2-specific IFN $\gamma$ response in splenocytes of BALB/c vaccinated mice, $n=6$ (G) ELISA detecting HER2-specific antibody production in serum of BALB/cvaccinated mice. $n=6(H)$ IFN $\gamma$ and TNF $\alpha$ production in CD8+ cells of BALB/c vaccinated mice, $n=6$. CD8+. T cells were gated on CD45+CD3+CD8+ cells. (I) IFN $\gamma$ and TNF $\alpha$ production in CD4+ cells of BALB/c vaccinated mice, $n=6$. CD4+ T cells were gated on CD45+CD3+CD4+ cells. ECD/ICD, extracellular and intracellular domains; HER2, human epidermal growth factor receptor 2; IFN $\gamma$, interferon- $\gamma$; LAMP, lysosome-associated membrane; OVA, ovalbumin; TNF $\alpha$, tumor necrosis factor $\alpha$. ANOVA. ${ }^{*} \mathrm{p}<0.05,{ }^{* *} \mathrm{p}<0.01$. 
A

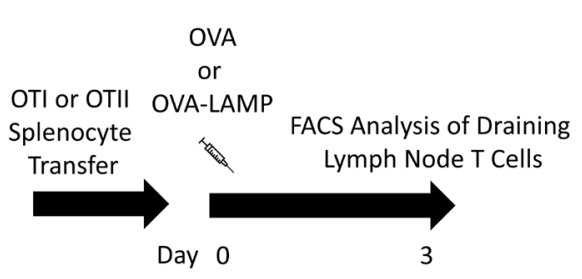

D

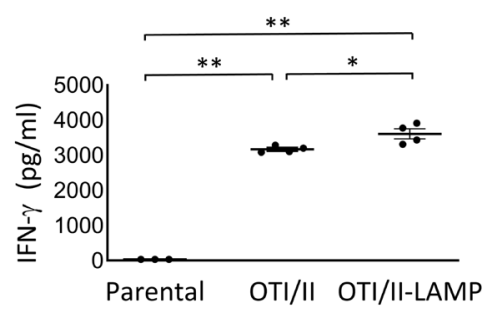

B

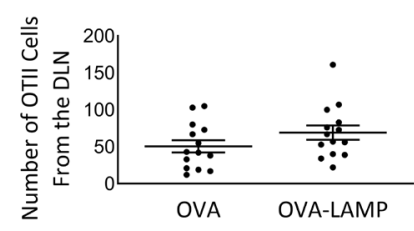

C

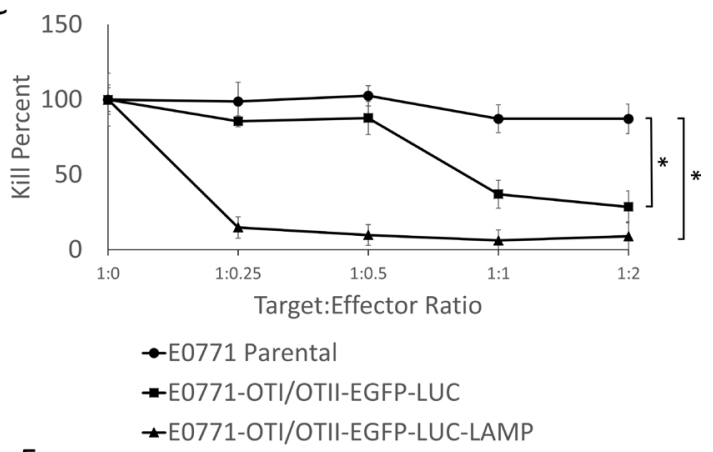

$\mathrm{F}$

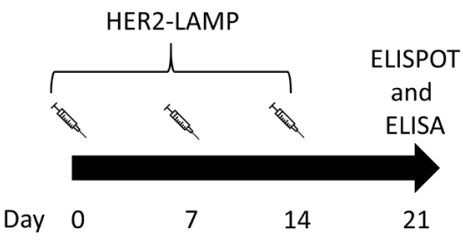

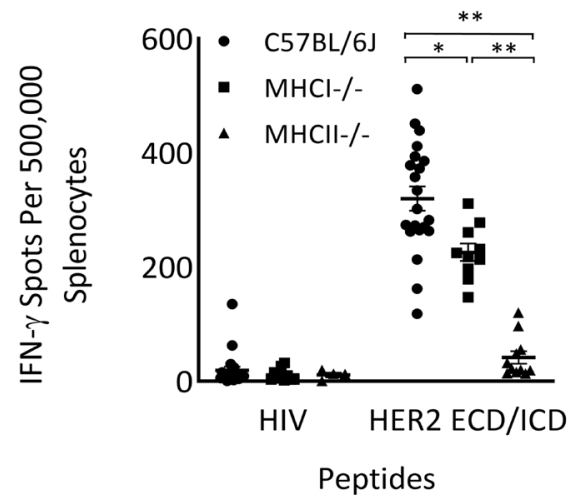

Figure 3 Antigen-specific T cell response elicted by LAMP-mediated antigen processing through the $\mathrm{MHCl}$ or $\mathrm{MHCll}$ antigen presentation pathways. OTI or OTII CD45.2 splenocytes were transferred into CD45.1 recipient mice. Recipient mice were vaccinated via intradermal electroporation with HER2-WT or HER2-LAMP. T cells were analyzed by FACS from draining lymph nodes (DLN) 3 days postvaccination. (B) Flow cytometric analysis of total number of OTIl cells (gated on CD45.2+ CD3+ and CD4+) in the DLNs, $n=14$ (C) Kill curve of E0771-OTI/OTII cells cocultured with OTI splenocytes, $n=4$ (D) ELISA detecting IFN $\gamma$ secretion into the supernatant of E0771-OTI/OTII cells cocultured with OTI splenocytes, $n=4$ (E) MHCl-/- or MHCII/- mice were vaccinated via intramuscular electroporation with $20 \mu \mathrm{g}$ HER2-LAMP with 2 homologous boosts administered at 7 and 14 days after the initial vaccination. ELISPOT and ELISA were performed 7 days after the last vaccination. (F) ELISPOT detecting HER2-specific IFN $\gamma$ response in splenocytes of vaccinated C57BL/6 control $n=22, \mathrm{MHCl} /-\mathrm{n}=11$, or MHCII-/- $n=11$ mice. HER2, human epidermal growth factor receptor 2; IFN $\gamma$, interferon- $\gamma$; LAMP, lysosome-associated membrane; OVA, ovalbumin; EGFP, enhanced green fluorescent protein; LUC, luciferase. ANOVA. ${ }^{*} p<0.05,{ }^{* *} p<0.01$.

the vaccinated mice were restimulated with HER2 ECD and ICD peptides, and IFN $\gamma$ production was measured by ELISPOT. HER2-LAMP vectors elicited significantly enhanced HER2-specific $\mathrm{T}$ cell responses (figure 2B), although not HER2-specific antibody responses to HER2 ECDs (online supplementary fig S2A). To confirm these enhanced responses for HER2 were not impacted by HER2 signaling from our control plasmid (containing full length HER2) or due solely to our specific type of electroporation, we again repeated an intramuscular electroporation strategy (using a higher intensity single vaccination) using HER2-ECD-transmembrane (HER2ECD-TM) and HER2-LAMP plasmids and assessed HER2-ECD-specific T cell and B cell responses. These experiments revealed that while HER2-ECD-TM could also elicit robust HER2-ECD-specific T-cell responses, HER2-LAMP again generated a significantly enhanced HER2-specific response (figure 2C). As before, we noted reduced HER2-specific antibody responses, suggesting that altering LAMP trafficking may have biased HER2specific immune responses towards Th1-type responses (online supplementary fig S2B). As these altered T cell responses were likely a mixture of class I and class II presented peptides, we next used our OTI/II-GFP plasmids to interrogate if we could specifically alter class I and II specific peptide responses, using peptide-specific ELISPOT assays. Using the same electroporation strategy, we found that OTI/II-EGFP-LAMP vectors allowed for significantly enhanced IFN $\gamma$ responses to SIINFEKL (OTI target) class I peptide and modestly improved IFN $\gamma$ responses from an ISQVHAAHAEINEAGR (OTII-target) class II peptide, suggesting that LAMP trafficking can enrich for IFN $\gamma$ responses against class I effector CD8+T cells (figure 2D). To further probe these differences, we used HER2-WT and HER2-LAMP plasmids with a homologous plasmid boosting strategy using intradermal electroporation to specifically enrich for DC targeting as previously described $^{31-34}$ (figure 2E). Using this strategy, we again found that HER2-LAMP vectors elicited significantly enhanced HER2-specific IFN $\gamma$ ELISPOT responses compared with HER2-WT (figure 2F), along with diminished HER2-specific antibody responses (figure 2G). To assess differences in levels of polyfunctional HER2specific $\mathrm{T}$ cells, we restimulated CD8+ and CD4+ T cells 


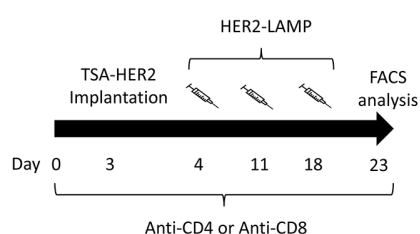

$\mathrm{D}$
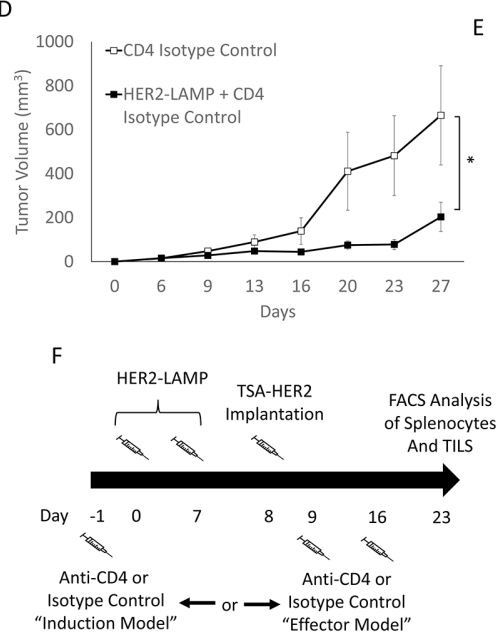

B
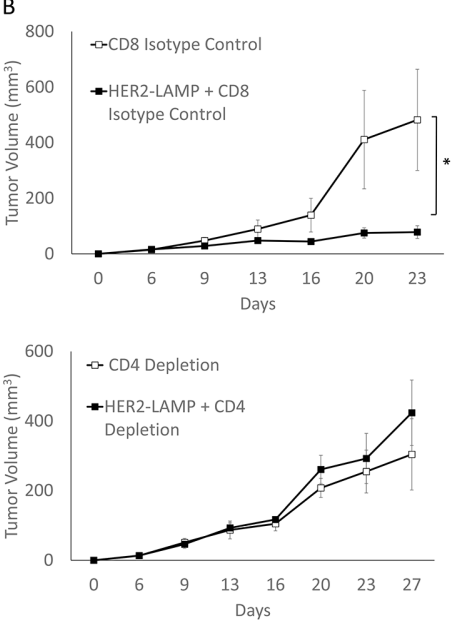

G

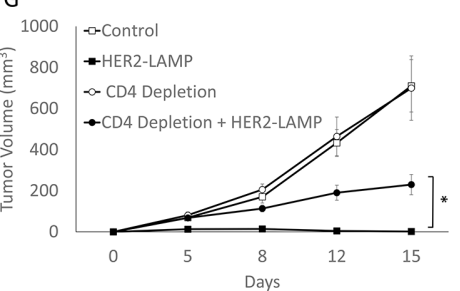

C

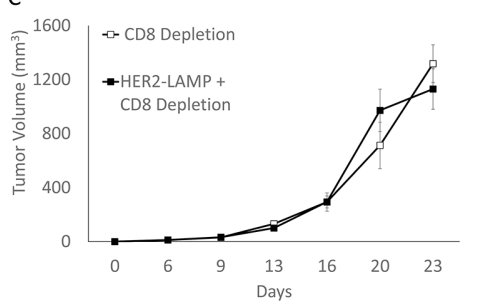

Figure 4 HER2-LAMP vaccination inhibits tumor growth in a CD4 and CD8-dependent manner. (A) BALB/c mice were administered with anti-CD4 or anti-CD8 antibodies to deplete their respective populations throughout this experiment, followed by implantation of 200,000 TSA-HER2 cells into the mammary fat pad. Intradermal electroporation was administered using 40 $\mu \mathrm{g}$ control vector or $40 \mu \mathrm{g}$ HER2-LAMP with 2 homologous boosts administered at 1, 7, and 14 days after transplantation. (B) Tumor growth of HER2-LAMP vaccinated, TSA-HER2 implanted mice during CD8 isotype control treatment, $n=6$ (C) Tumor growth of HER2-LAMP vaccinated, TSA-HER2 implanted mice during CD8 depletion, $n=6$ (D) Tumor growth of HER2-LAMP vaccinated, TSA-HER2 implanted mice during CD4 isotype control treatment, $n=6$ (E) Tumor growth of HER2-LAMP vaccinated, TSA-HER2 implanted mice during CD4 depletion, $n=3$ (F) BALB/c mice wereadministered with implanted with 200,000 TSAHER2 cells into the mammary fat pad, and vaccinated via intradermal electroporation with 20 ug of HER2-LAMP twice. AntiCD4 depletion antibody was injected prior to vaccination (induction model) or after implantation (effector model). (G) Tumor growth of HER2-LAMP vaccinated mice in the induction model. $n=7(H)$ Tumor growth of HER2-LAMP vaccinated mice in the effector model. $n=7$. HER2, human epidermal growth factor receptor 2; LAMP, lysosome-associated membrane. ANOVA. ${ }^{*} \mathrm{p}<0.05,{ }^{* *} \mathrm{p}<0.01$.

from vaccinated mice with HER2 peptides and stained for intracellular IFN $\gamma$ and TNF $\alpha$. These experiments revealed that while both vaccines could stimulate CD8+ and CD4+ HER2-specific cytokine responses, HER2-LAMP vectors elicited greater quantities of IFN $\gamma / \mathrm{TNF} \alpha$ doubleproducing CD8+ T cells (figure $2 \mathrm{H}$ ), but had no effect on $\mathrm{CD} 4+\mathrm{T}$ cell cytokine production at day 28 postvaccination (figure 2I). These data demonstrate that vaccination with LAMP trafficking vectors results in enhanced antigen-specific CD8+ T cell responses.

\section{Importance of both MHC class I and II presentation in generating antigen-specific $\mathrm{T}$ cell responses from lamp vectors in vitro and in vivo}

As our data indicated that LAMP trafficking could enhance the quantity and quality of $\mathrm{CD} 8+\mathrm{T}$ cell responses, we next tested if these events were potentially due to early activation of CD8+ and CD4+ helper T cells. To determine the activation of $\mathrm{T}$ cells in this system, we injected a limited number of congenic class I-restricted CD8+ OTI and class II restricted CD4+ OTII cells (labeled with carboxyfluorescein succinimidyl ester (CSFE) ) into C57BL/6 mice and then vaccinated mice with OVA-WT and OVA-LAMP vectors using intradermal electroporation (figure $3 \mathrm{~A}$ ). Using this strategy, we isolated the draining lymph nodes at an early time point (3 days postvaccination) to assess how these vaccines affected the early expansion $\left(\mathrm{CSFE}^{\mathrm{lo}}\right)$ and activation $\left(\mathrm{CD} 44^{\mathrm{hi}}\right)$ of OVA-specific $\mathrm{T}$ cells. These experiments revealed a greater number of activated and expanded CD4+ OTII cells from OVA-LAMP vaccination in comparison to OVA-WT control vaccination (figure 3B-C). This suggested that altered OVA trafficking in sentinel cells leads to enhanced $\mathrm{T}$ cell stimulation, presumably through enhanced class II presentation.

While previous studies have documented LAMP trafficking to enhance class II peptide presentation, ${ }^{11-13} 23$ we wanted to determine if LAMP could also directly alter class I peptide presentation. To determine class I presentation, we tested the ability of LAMP or control vectors to present SIINFEKL peptides to OTI-specific T cells from non-professional antigen presenting tumor cells. We stably transduced $\mathrm{E} 0771$ cells (a C57BL/6 BC line) with OTI/II-EGFP or OTI/II-EGFP-LAMP lentiviral vectors 

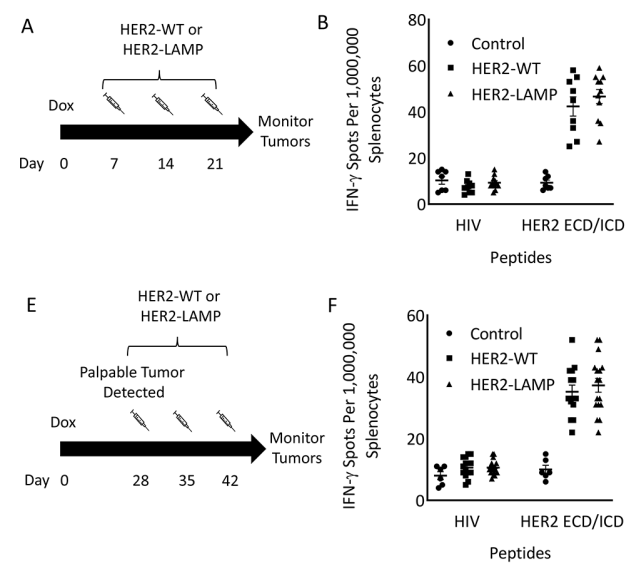

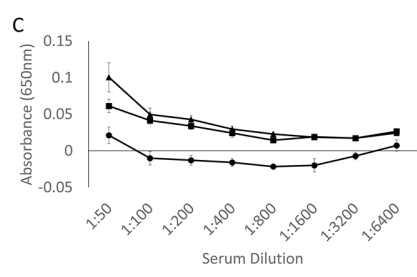

$\rightarrow$ Control - HER2-WT $\rightarrow$ HER2-LAMP

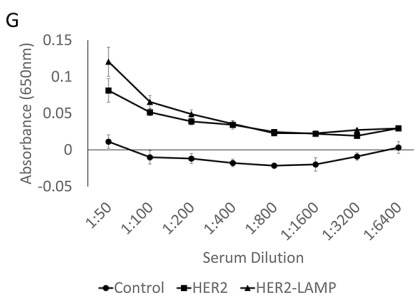

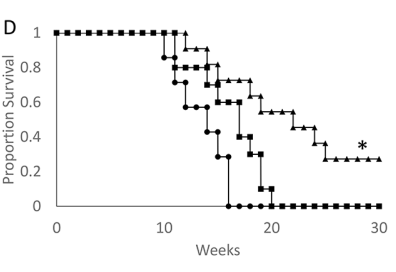

$\bullet$ Control - HER2-WT $\leftarrow$ HER2-LAMP

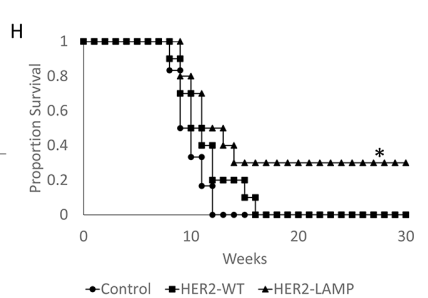

Figure 5 Early and late therapeutic HER2-LAMP vaccinations in a spontaneous HER2+ breast cancer model. (A) HER2 $\triangle 16$ mice were vaccinated via intramuscular electroporation with $20 \mu \mathrm{g}$ of OVA-LAMP (control) $n=7,20 \mu \mathrm{g} \mathrm{HER}$-WT n=9 or $20 \mu \mathrm{g}$ HER2-LAMP $n=117$ days after doxycycline induction. Two homologous boosts were administered at 7 and 14 days after the initial vaccination. (B) ELISPOT to detect HER2-specific IFN- $\gamma$ production in splenocytes of HER2 $\triangle 16$ vaccinated mice in the early therapeutic model. (C) Cell-based ELISA to detect HER2-specific antibodies in the serum ofHER2 $\triangle 16$ vaccinated mice in the early therapeutic model. (D) Survival curve of HER2 $\triangle 16$ vaccinated mice in the early therapeutic model. (E) HER2 $\triangle 16$ mice were vaccinated via intramuscular electroporation with $20 \mu \mathrm{g}$ of OVA-LAMP (control) $\mathrm{n}=6,20 \mu \mathrm{g}$ HER-WT $\mathrm{n}=10$, or $20 \mu \mathrm{g}$ HER2-LAMP $n=10,28$ days after doxycycline induction upon the detection of the earliest palpable tumor. Two homologous boosts were administered at 7 and 14 days after the initial vaccination. (F) ELISPOT to detect HER2-specific IFN- $\gamma$ production in splenocytes of HER2 16 vaccinated mice in the late therapeutic model. (G) Cell-based ELISA to detect HER2-specific antibodies in the serum of HER2 $\Delta 16$ vaccinated mice in the late therapeutic model. $(H)$ Survival curve of HER2 $\Delta 16$ vaccinated mice in the late therapeutic model. ECD/ICD, extracellular and intracellular domains; HER2, human epidermal growth factor receptor 2; IFN $\gamma$, interferon- $\gamma$; LAMP, Iysosome-associated membrane; OVA, ovalbumin. ANOVA. ${ }^{*} \mathrm{p}<0.05,{ }^{* *} \mathrm{p}<0.01$.

and performed coculture experiments with OTI CD8+ T cells. As before, we found that OTI/II-EGFP had significantly elevated expression of EGFP compared with OTI/ II-EGFP-LAMP (online supplementary fig S3). Despite this expression difference, OTI CD8+ Tcell coculturing experiments demonstrated significantly greater lysis of OTI/II-EGFP-LAMP expressing cells in comparison to controls, consistent with enhanced presentation of SIINFEKL through the class I pathway (figure 3C). Notably, we also observed enhanced secretion of IFN $\gamma$ from these $\mathrm{T}$ cells, similar to what we had observed by ELISPOT in vaccinated mice (figure 3D). These studies suggested that enhanced class I presentation may also play a key role in LAMP-based vaccine efficacy. To formally test the contribution of class I and class II pathways on HER2-LAMP vaccine efficacy, we vaccinated mice deficient for class I $(\beta \mathrm{M} \mathrm{KO})$ and class II (MHCII KO) presentation pathways with HER2-LAMP in comparison to wild-type controls (figure 3E). These experiments demonstrated a modest, although significant reduction of HER2-specific $\mathrm{T}$ cell responses in MHCI KO mice, but ablated HER2-specific T cell and B cell responses in MHCII KO mice (figure 3F). This indicated that both pathways play roles in the induction of HER2-LAMP adaptive T cell responses, but that HER2-LAMP presentation through the MHC class II pathway is essential for HER2-specific T cell responses.

\section{HER2-LAMP efficacy depends upon both CD4+ and CD8+ T cells and elicits enhanced survival in a HER2+ BC model}

Having demonstrated enhanced induction of antigenspecific $\mathrm{T}$ cell immunity, we next wanted to assess if
HER2-LAMP vaccination could be an effective therapy against HER2+ BC in vivo and determine if these responses were dependent on CD8+ or CD4+ T cells. To test this, we orthotopically implanted wild-type HER2-expressing TSA cells into the mammary fat pad of BALB/c mice and vaccinated with HER2-LAMP plasmid electroporation 1 day postimplantation (figure $4 \mathrm{~A}$ ). To determine the effect of $\mathrm{CD} 8+$ and $\mathrm{CD} 4+\mathrm{T}$ cells, we administered control, $\mathrm{CD} 8$ or $\mathrm{CD} 4$ depleting antibodies prior to tumor implantation, maintaining a depletion regimen throughout the experiment. These studies revealed elimination of CD8+ T cells abrogated all antitumor responses from HER2-LAMP vaccination (figure 4B-C), suggesting that HER2-LAMP vaccination efficacy is directly mediated by $\mathrm{CD} 8+\mathrm{T}$ cells. Additionally, we found that depletion of CD4+ T cells eliminated the antitumor effect of the HER2-LAMP vaccine (figure 4D-E), suggesting that HER2-LAMP vaccination efficacy is also directly mediated by $\mathrm{CD} 4+\mathrm{T}$ cells. To address if $\mathrm{CD} 4+\mathrm{T}$ cells are critical to the induction of HER2-LAMP vaccine responses, we administered control or CD4 depleting antibodies prior to vaccination and TSA-HER2 tumor challenge (figure 4F, online supplementary fig S4). These studies revealed that tumor growth was only partially inhibited by the HER2-LAMP vaccine after CD4 depletion, indicating that $\mathrm{CD} 4+\mathrm{T}$ cells play an important role in the induction phase of the immune response (figure 4G). As in non-tumor bearing mice, we again observed that HER2LAMP vaccination significantly augmented the activation of CD8+ HER2-specific T-cells, which associated with 

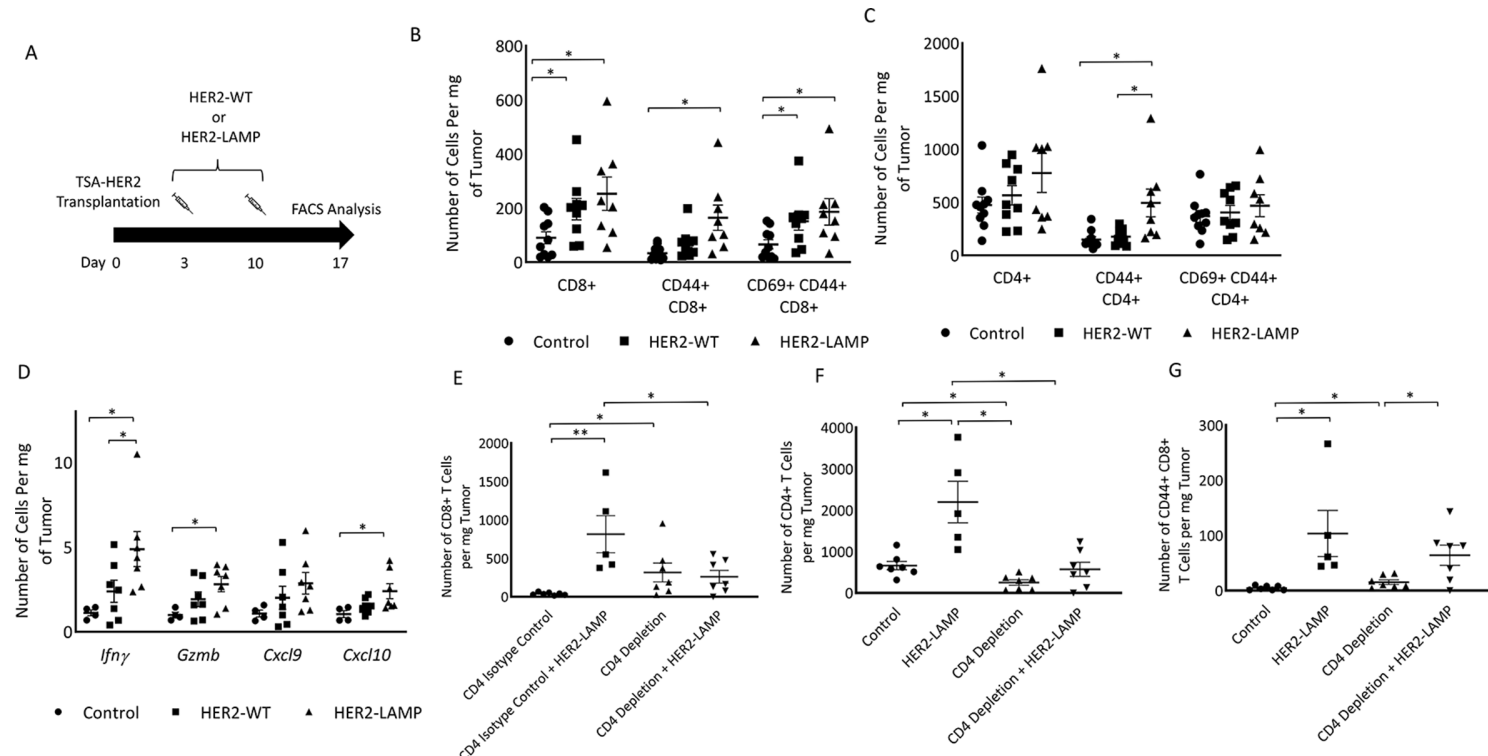

Figure 6 HER2-LAMP alters tumor infiltration and microenvironment in a orthotopic model. (A) BALB/c mice were implanted with 100,000 TSA-HER2 cells into the mammary fat pad. Intradermal electroporation was administered using $40 \mu \mathrm{g}$ control vector $n=10,40 \mu \mathrm{g}$ HER2-WT $n=9$, or $40 \mu \mathrm{g}$ HER2-LAMP $\mathrm{n}=8$ with two immunizations administered at 3 and 10 days after transplantation. FACS analyses on TILS were performed 7 days after the last vaccination. (B) Amount of CD8+ tumor infiltrating lymphocytes isolated from tumors of vaccinated mice. (C) Amount of CD4+ tumor infiltrating lymphocytes isolated from tumors of vaccinated mice. (D) The expression of IFNg, Gzmb, and Cxcl10 in the tumors of vaccinated mice as determined by qRTPCR. $n=4-7$ (E) Total number of CD8+, CD4+ (F), and CD44+/CD8+ T cell infiltrates in the tumors of HER2-LAMP vaccinated mice used in the induction model. $n=5-7$. HER2, human epidermal growth factor receptor 2 ; IFN $\gamma$, interferon- $\gamma$; LAMP, Iysosomeassociated membrane. ANOVA. ${ }^{*} \mathrm{p}<0.05,{ }^{* *} \mathrm{p}<0.01$.

antitumor responses (online supplementary fig S5A-C), but not the percentage of systemic activated CD4+ T cells (online supplementary fig S5D). To address the role of CD4+ T cells in the effector phase of HER2-LAMP vaccine induced antitumor responses, we administered control or CD4 depleting antibodies postvaccination and TSA-HER2 tumor challenge (figure $4 \mathrm{~F}$ ). These studies again revealed that CD4 depletion at this phase had no significant effect on HER2-LAMP mediated antitumor responses. Taken together these results demonstrate that $\mathrm{CD} 4+\mathrm{T}$ cells have essential function in the induction phase, but not the effector phase of HER2-LAMP vaccine driven antitumor immunity.

HER2-LAMP vaccination enhances survival in both early and late stage HER2+ BC, potentially mediated by enhanced CD4+ and CD8+ T cell activation in the tumor microenvironment (TME).

Having demonstrated an antitumor effect from HER2LAMP vaccination dependent on both CD $4+$ and CD8+T cells, we next wanted to test whether HER2-LAMP could provide superior antitumor efficacy compared with a HER2-WT vaccine. To answer this, we used an endogenous model of HER2+ BC that is tolerant to HER2, dependent on HER2 expression/signaling (in contrast to TSA-HER2 ${ }^{35}$ and develops metastatic HER2+ BC (approximately $90 \%$ of mice bear lung metastases), resistant to late stage therapeutic modalities (T-DM1). ${ }^{27}$ This model allowed us to test the efficacy of vaccines at different time points in developing spontaneous HER2 $+\mathrm{BC}$. To test the impact of vaccines in early stage HER2+ BC, HER2 216 was induced by doxycycline administration and mice were vaccinated 1 week postinduction to simulate HER2 responses against developing HER2+ BC (figure 5A). Significantly, in this model both HER2-WT and HER2LAMP vaccines overcame HER2 tolerance and induced HER2-specific systemic $\mathrm{T}$ cell and $\mathrm{B}$ cell responses (figure 5B-C), with a trend of $\mathrm{T}$ cell and $\mathrm{B}$ cell responses being greater in HER2-LAMP vaccinated mice. Strikingly, mice vaccinated with HER2-LAMP showed a significant survival advantage over mice vaccinated with HER2-WT (figure 5D). Notably, HER2-LAMP-vaccinated tumor-free mice displayed similar HER2-specific systemic T cell and B cell responses in comparison to tumor-bearing vaccinated mice (online supplementary fig S6A). This suggested that the survival difference may be mediated through altered trafficking and activation of T cells within the TME.

As our past studies and those of others have demonstrated that established TMEs are highly immunosuppressive, ${ }^{36-39}$ we next wanted to test the potential of HER2-LAMP vaccines in more established tumors. Taking advantage of our endogenous model, we again elicited HER2 $\Delta 16$ expression using doxycycline but did not begin vaccination until the first tumor reached a volume of $\sim 100 \mathrm{~mm}^{3}$ (figure 5E). At this time, tumor-bearing mice were randomly enrolled into control vaccine (OVALAMP), HER2-WT or HER2-LAMP plasmid vaccine treatment groups. As before, we found that HER2-specific $\mathrm{T}$ cell responses were present in all HER2-vaccinated mice (figure $5 \mathrm{~F}-\mathrm{G}$ ), but did not differ between vaccine groups or between tumor-free and tumor-bearing animals 
(figure 6B). However, we again found that mice vaccinated with HER2-LAMP demonstrated a significant survival advantage over the mice vaccinated with HER2WT, even in mice with palpable tumors (figure $5 \mathrm{H}$ ), signifying that HER2-LAMP is an effective vaccine when administered as a therapeutic agent in established HER2 +disease. Collectively, the systemic responses at these later time points ( 9-30 weeks postvaccination) did not differ between the HER2-WT and HER2-LAMP cohorts, suggesting that differences in HER2-LAMP-elicited T cells may occur after the early induction of responses and their initial infiltration into these heterogeneous TMEs.

As our data indicated potential differences in a subset of endogenous tumors that regressed after HER2-LAMP vaccination, we elected to test the infiltration of $\mathrm{T}$ cells at earlier points postvaccination in a more homogeneous orthotopic TSA-HER2 model. Mice were implanted with tumors and vaccinated with control, HER2-WT or HER2LAMP vectors (at day 3 and 10), before being sacrificed at day 17 to assess potential differences in infiltrating $\mathrm{T}$ cells (figure $6 \mathrm{~A}$ ). We found that while both vaccines significantly augmented CD8+ and CD4+ Tcell infiltration, HER2-LAMP elicited significantly higher numbers of CD44+CD4+ T cells in comparison to HER2-WT vaccination (figure $6 \mathrm{~B}-\mathrm{C}$ ). Moreover, tumors from HER2LAMP-vaccinated mice expressed higher levels of $\mathrm{T}$ cell effector genes, such as Ifng and $G z m b$, as well as the T cell trafficking chemokine Cxcl10, compared with HER2-WT controls (figure 6D). To determine if these inductions were due to enhanced CD4 +Tcell 'help' during the induction phase, we assessed tumor infiltrates from mice that had been CD4 depleted prior to vaccination, along with respective controls (figure $4 \mathrm{~F}-\mathrm{G}$ ). These assessments revealed that HER2-LAMP vaccination significantly augmented the abundance of total tumor-infiltrating CD8+ and CD4+ Tcells (figure 6E-F), as well as CD44/ CD8+ Tcells in tumors compared with control mice (figure 6G). Notably, depleting CD4 +T cells during the induction phase decreased CD8 + Tcell infiltration, as well as CD4+ T cells, in tumors of HER2-LAMP vaccinated mice (figure 6E-F). Additionally, we confirmed elevated expression of IFN $\gamma$, Granzyme B, CXCL9 and CCL3 by qRT-PCR in these samples (online supplementary fig S7A-D). Consistent with these observations, vaccination with HER2-LAMP in a separate set of experiments using the orthotopic TSA-HER2 model (online supplementary fig S7E) also elicited Ifng and $\mathrm{Gzmb}$, as well $\mathrm{Ccl3}, \mathrm{Cxcl} 9$ and Cxcl10 compared with HER2-WT controls (online supplementary fig S7F-J). Collectively, these studies suggest that LAMP trafficking elicits robust activation and expansion of antigen-specific T cells, partially dependent on CD4+ $\mathrm{T}$ cells in the induction phase of vaccination, that may confer an antitumor survival advantage in the TME.

\section{DISCUSSION}

In this study, we found that conjugation of different antigens to LAMP led to enhanced trafficking to endolysosomal subcellular compartments, which associated with significantly decreased levels of protein expression across multiple antigens and in multiple cell types (Figure 1, online supplementary S1). However, despite a reduction in overall antigen expression, HER2-LAMP vaccines allowed for enhanced antigen-specific $\mathrm{T}$ cell responses to HER2 epitopes (encompassing both the ECD and the ICD domains) in comparison to control HER2 vectors in vivo. These enhanced responses were unaltered by different types of plasmid vaccination strategies (intramuscular or intradermal) and resulted in greater numbers of polyfunctional HER2-specific CD8+ effector T cells by HER2-LAMP vaccines in comparison to HER2-WT vaccines. Studies in infectious disease have demonstrated that vaccines capable of producing polyfunctional $\mathrm{T}$ cell responses are highly effective, which emerging immunooncology studies have likewise suggested may be critical for effective anti-tumor immunity. ${ }^{40-45}$ To investigate how LAMP trafficking may generate these enhanced responses, we focused on potential differences in MHC class I and II presentation and resulting CD4+ and CD8+ Tcell activation.

Previous studies have demonstrated that LAMP vectors can enhance MHC class II presentation and CD4+ Tcell responses, ${ }^{1322-2430} 46$ which we likewise observe through enhanced activation of OTII-specific CD4+ T cells after OVA-LAMP vaccination in comparison to OVA-WT vaccination (figure 3B). Using MHCII KO mice, we also found that HER2-LAMP vaccine presentation of HER2 epitopes through MHC class II was essential for induced HER2specific $\mathrm{T}$ cell responses, suggesting a critical role for MHCII presentation in HER2-LAMP vaccine-induced HER2-specific immunity. This effect was validated in $\mathrm{T}$ cell depletion experiments that revealed that $\mathrm{CD} 4+$ $\mathrm{T}$ cells played an important role in mediating HER2LAMP antitumor immunity in the induction of immune responses from HER2-LAMP vaccination, but not in the antitumor effector phase (figure 4). Additionally, our studies also noted a direct enhancement of MHC class I presentation through enhanced stimulation and direct lysis of OTI/II-EGFP-LAMP expressing tumor cells by OTI CD8+ T cells in vitro, as well as enhanced expansion and activation of OTI T-cells after OVA-LAMP vaccination in comparison to OVA-WT vaccination in vivo. While the use of MHCI KO mice demonstrated suppressed HER2-specific $T$ cell responses after HER2-LAMP vaccination, we found that CD8+ T cells were essential for HER2-LAMP anti-tumor immune responses. These data, along with our MHCII findings, suggest that HER2-LAMP enhances presentation and stimulates direct activation of CD8+ T cells, but also enhances MHCII presentation, in order to more effectively activate CD4+ T cells. In combination, the enhanced activation of class I and II pathways generate superior polyfunctional CD8+ Tcell responses, which are able to more effectively infiltrate the TME (potentially through enhanced chemokine secretion) and elicit tumor-specific killing. Our depletion studies indicate CD8+ T cells are the dominant effector cells of 
HER2-LAMP vaccine antitumor responses, with HER2specific antibodies playing a minor role in observed antitumor responses.

Perhaps most importantly, our studies also demonstrated that HER2-LAMP vaccination elicits long-term survival and complete tumor regression in $\sim 30 \%$ of mice after both early and late vaccination in an aggressive and targeted therapy-resistant endogenous model of HER2+ BC. This result was striking, as our previous studies have demonstrated greater difficulty in immunotherapeutic strategies to elicit curative immunity in advanced cancers. ${ }^{63947}$ Notably, all vaccinated mice demonstrated significant systemic induction of HER2-specific T cell and $\mathrm{B}$ cell responses after vaccination that was similar between mice that remained tumor-free and those that had progressive HER2+ disease. This suggested that the differences in vaccine-induced antitumor immunity could be due to early differences in the induction of immunity that translated into differences in the TME. Using an orthotopic model that allowed us to examine tumors earlier postvaccination, we confirmed that HER2-LAMP vaccination induced more activated CD8+ and CD4+ Tcell populations in the TME, as well as higher expression of IFN $\gamma$, Granzyme B and various chemokines. These CD8+ Tcell infiltrates in the TME were suppressed by depletion of CD4+ T cells during HER2-LAMP vaccination. This again suggests that HER2-LAMP-augmented antitumor immunity and increased survival may be due to a more potent activation of helper and subsequent activation of effector $\mathrm{T}$ cells that induce effector genes and recruitment chemokine profiles, which would be capable of causing tumor regression in a significant fraction of cancers. This response rate is similar to that seen in many cancers for PD-1/PD-L1 immune checkpoint blockade, where $\sim 20 \%$ of mice respond to treatment. Notably, we have found that the use of PD-1 mAbs in this model has 100\% mortality rate by 18 weeks (Crosby et al, Clinical Cancer Research (CCR) in review). This suggests that HER2LAMP vaccination may offer a compelling approach to elicit anti-tumor immunity in aggressive cancers with low neoepitope burdens.

Collectively, these results suggest that LAMP-targeted vectors allow for enhanced endolysosomal antigen targeting which precipitates enhanced MHC class I and II presentation. This enhanced presentation results in greater activation of both $\mathrm{CD} 4+$ and CD8+ Tcell responses, polyfunctional CD8 $+\mathrm{T}$ cells, and ultimately tumor rejection and long-term survival in a subset of mice. This mirrors preclinical and clinical studies with different immunotherapies in BC that demonstrate heterogeneous tumor regression associated with differences in $\mathrm{T}$ cell infiltration and local immunosuppression. ${ }^{39} 4849$ As our clinical studies suggest that the stimulation of HER2-specific memory CD8+ T cells is an important requirement for progression-free survival, ${ }^{6}$ the use of HER2-LAMP vaccines may have a significant impact against treatment-resistant metastatic HER2+ BC. Given that effective local immunosuppression can still curtail these responses in a significant fraction of mice, HER2-LAMP vaccines may be effectively coupled with heterologous vaccines (such as our viral HER2 vectors) to further expand anti-HER2 immune responses, or with PD-1/PD-L1 immune checkpoint inhibitors to counterbalance local immunosuppression, which are the focus of our continuing investigations. Additionally, the ability to elicit potent antitumor immunity against self-antigens suggests that this strategy may be highly effective in generating antitumor responses. Indeed, several studies have suggested that LAMP targeting allows for more effective HPV E6/E7-specific immune responses, as well as for enhanced antitumor immunity against HPV-related cancers. ${ }^{11} 29305051$ More broadly, this study suggests that LAMP-based targeted therapies may be highly effective in the generation of novel cancer vaccines against overexpressed or mutated endogenous oncogenes in order to elicit robust, multipotent CD8+ Tcell responses against established targeted-therapy resistant tumors.

\section{Author affiliations}

${ }^{1}$ Department of Surgery, Duke University, Duke University, Durham, North Carolina, USA

${ }^{2}$ Department of R\&D, Immunomic Therapeutics, Rockville, Maryland, USA ${ }^{3}$ Department of Pathology, Duke University, Durham, North Carolina, USA

${ }^{4}$ Department of Immunology, Duke University, Durham, NC, USA

Acknowledgements We would also like to acknowledge all of the members of the Applied Therapeutics Center, and Dr. Mohammad Ariful Islam and Dr. Claire Rosean from Immunomic Therapeutics (ITI) for their generous technical assistance, help and insights into this project.

Contributors $\mathrm{ACC}$ and $\mathrm{RX}$ have contributed equally to the paper as first coauthors. $\mathrm{ZCH}, \mathrm{ACC}$ and $\mathrm{RX}$ wrote the manuscript. $\mathrm{ZCH}, \mathrm{HKL}$ and TH provided supervision to the project. TW performed flow cytometry and analysis. JW, XY, C-XL and GL assisted in animal procedures and in vitro studies.

Funding This research was supported by grants from the National Institutes of Health (NIH) (5K12CA100639-09 to ZCH, 1R01CA238217-01A1 to ZCH) and (T32CA211056-01A1 to AC), Department of Defense (DOD) (BC113107 to HKL) and Susan G Komen (CCR14299200 to ZCH), as well as support from ITI.

Competing interests This work was supported in part by Immunomic Therapeutics. RX and TH are employees of Immunomic Therapeutics, and TH is a shareholder of Immunomic Therapeutics.

Patient consent for publication Not required.

Ethics approval All experimental procedures involving mice were approved by Duke University's Animal Care and Use Program which is fully accredited by the Association for the Assessment and Accreditation of Laboratory Animal Care.

Provenance and peer review Not commissioned; externally peer reviewed.

Data availability statement All data relevant to the study are included in the article or uploaded as on;ine supplementary information. All data are included in the article. Any clarifications on data, protocols or statistics will be available on request.

Open access This is an open access article distributed in accordance with the Creative Commons Attribution Non Commercial (CC BY-NC 4.0) license, which permits others to distribute, remix, adapt, build upon this work non-commercially, and license their derivative works on different terms, provided the original work is properly cited, appropriate credit is given, any changes made indicated, and the use is non-commercial. See http://creativecommons.org/licenses/by-nc/4.0/.

\section{ORCID iD}

Zachary Conrad Hartman http://orcid.org/0000-0001-6549-8207 


\section{REFERENCES}

1 Martin M, López-Tarruella S. Emerging therapeutic options for HER2-positive breast cancer. Am Soc Clin Oncol Educ Book 2016;35:e64-70.

2 Moasser MM, Krop IE. The evolving landscape of HER2 targeting in breast cancer. JAMA Oncol 2015;1:1154-61.

3 Yan M, Schwaederle M, Arguello D, et al. Her2 expression status in diverse cancers: review of results from 37,992 patients. Cancer Metastasis Rev 2015;34:157-64.

4 Laakmann E, Müller V, Schmidt M, et al. Systemic treatment options for HER2-positive breast cancer patients with brain metastases beyond trastuzumab: a literature review. Breast Care 2017;12:168-71.

5 Nuciforo P, Radosevic-Robin N, Ng T, et al. Quantification of her family receptors in breast cancer. Breast Cancer Res 2015;17:53.

6 Crosby EJ, Gwin W, Blackwell K, et al. Vaccine-Induced Memory $\mathrm{CD}^{+} \mathrm{T}$ Cells Provide Clinical Benefit in HER2 Expressing Breast Cancer: A Mouse to Human Translational Study. Clin Cancer Res 2019;25:2725-36.

7 Hartman ZC, Wei J, Osada T, et al. An adenoviral vaccine encoding full-length inactivated human HER2 exhibits potent immunogenicty and enhanced therapeutic efficacy without oncogenicity. Clin Cancer Res 2010;16:1466-77.

8 Hartman ZC, Wei J, Glass OK, et al. Increasing vaccine potency through exosome antigen targeting. Vaccine 2011;29:9361-7.

9 Kasturi SP, Pulendran B. Cross-Presentation: avoiding trafficking chaos? Nat Immunol 2008;9:461-3.

10 Kreiter S, Selmi A, Diken M, et al. Increased antigen presentation efficiency by coupling antigens to MHC class I trafficking signals. J Immunol 2008;180:309-18.

11 Wu TC, Guarnieri FG, Staveley-O'Carroll KF, et al. Engineering an intracellular pathway for major histocompatibility complex class II presentation of antigens. Proc Natl Acad Sci U S A 1995;92:11671-5.

12 Rowell JF, Ruff AL, Guarnieri FG, et al. Lysosome-Associated membrane protein-1-mediated targeting of the HIV-1 envelope protein to an endosomal/lysosomal compartment enhances its presentation to MHC class II-restricted T cells. Journal of immunology 1995;155:1818-28.

13 Fernandes DM, Vidard L, Rock KL. Characterization of MHC class II-presented peptides generated from an antigen targeted to different endocytic compartments. Eur J Immunol 2000;30:2333-43.

14 Kreiter S, Vormehr M, van de Roemer N, et al. Mutant MHC class II epitopes drive therapeutic immune responses to cancer. Nature 2015;520:692-6.

15 Kodumudi KN, Ramamoorthi G, Snyder C, et al. Sequential anti-PD1 therapy following dendritic cell vaccination improves survival in a HER2 mammary carcinoma model and identifies a critical role for CD4 T cells in mediating the response. Front Immunol 1939;2019:10.

16 Nocera NF, Lee MC, Czerniecki BJ. Boosting anti-HER2 CD4 T-helper responses in HER2 expressing ductal carcinoma in situ. Future Oncol 2017;13:1459-62.

17 De La Cruz LM, McDonald ES, Mick R, et al. Anti-HER2 CD4 ${ }^{+}$ T-Helper Type 1 Immune Response is Superior to Breast MRI for Assessing Response to Neoadjuvant Therapy in Patients with HER2Positive Breast Cancer. Ann Surg Oncol 2017;24:1057-63.

18 Datta J, Berk E, Xu S, et al. Anti-HER2 CD4(+) T-helper type 1 response is a novel immune correlate to pathologic response following neoadjuvant therapy in HER2-positive breast cancer. Breast Cancer Res 2015;17:71.

19 Jiang D-B, Sun L-J, Cheng L-F, et al. Recombinant DNA vaccine of hantavirus GN and LAMP1 induced long-term immune protection in mice. Antiviral Res 2017;138:32-9.

20 Rigato PO, Maciel M, Goldoni AL, et al. Immunization of neonatal mice with LAMP/p55 HIV gag DNA elicits robust immune responses that last to adulthood. Virology 2010;406:37-47.

21 Yang K, Sun K, Srinivasan KN, et al. Immune responses to T-cell epitopes of SARS CoV-N protein are enhanced by $\mathrm{N}$ immunization with a chimera of lysosome-associated membrane protein. Gene Ther 2009;16:1353-62.

22 Anwar A, Chandrasekaran A, Ng ML, et al. West Nile premembraneenvelope genetic vaccine encoded as a chimera containing the transmembrane and cytoplasmic domains of a lysosomeassociated membrane protein: increased cellular concentration of the transgene product, targeting to the MHC II compartment, and enhanced neutralizing antibody response. Virology 2005;332:66-77.

23 Marques ETA, Chikhlikar P, de Arruda LB, et al. Hiv-1 p55Gag encoded in the lysosome-associated membrane protein-1 as a DNA plasmid vaccine chimera is highly expressed, traffics to the major histocompatibility class II compartment, and elicits enhanced immune responses. J Biol Chem 2003;278:37926-36.
24 Lu Y, Raviprakash K, Leao IC, et al. Dengue 2 PreM-E/LAMP chimera targeted to the $\mathrm{MHC}$ class II compartment elicits long-lasting neutralizing antibodies. Vaccine 2003;21:2178-89.

25 Su Y, Romeu-Bonilla E, Anagnostou A, et al. Safety and long-term immunological effects of CryJ2-LAMP plasmid vaccine in Japanese red cedar atopic subjects: a phase I study. Hum Vaccin Immunother 2017;13:2804-13.

26 Su Y, Connolly M, Marketon A, et al. CryJ-LAMP DNA vaccines for Japanese red cedar allergy induce robust Th1-type immune responses in murine model. J Immunol Res 2016;2016:1-15.

27 Turpin J, Ling C, Crosby EJ, et al. The ErbB2 $\Delta$ Ex16 splice variant is a major oncogenic driver in breast cancer that promotes a prometastatic tumor microenvironment. Oncogene 2016;35:6053-64.

28 Starodubova E, Boberg A, Ivanov A, et al. Potent cross-reactive immune response against the wild-type and drug-resistant forms of HIV reverse transcriptase after the chimeric gene immunization. Vaccine 2010;28:1975-86.

$29 \mathrm{Kim}$ TW, Lee J-H, He L, et al. Dna vaccines employing intracellular targeting strategies and a strategy to prolong dendritic cell life generate a higher number of CD8+ memory T cells and better longterm antitumor effects compared with a DNA prime-vaccinia boost regimen. Hum Gene Ther 2005;16:26-34.

30 Lin KY, Guarnieri FG, Staveley-O'Carroll KF, et al. Treatment of established tumors with a novel vaccine that enhances major histocompatibility class II presentation of tumor antigen. Cancer Res 1996;56:21-6.

31 Schultheis K, Smith TRF, Kiosses WB, et al. Delineating the cellular mechanisms associated with skin electroporation. Hum Gene Ther Methods 2018;29:177-88.

32 Engelke L, Winter G, Hook S, et al. Recent insights into cutaneous immunization: how to vaccinate via the skin. Vaccine 2015;33:4663-74.

33 Lee S-H, Danishmalik SN, Sin J-I. Dna vaccines, electroporation and their applications in cancer treatment. Hum Vaccin Immunother 2015;11:1889-900.

34 Peachman KK, Rao M, Alving CR. Immunization with DNA through the skin. Methods 2003;31:232-42.

35 Xu R, Heiland T. Abstract LB-204: DNA vaccine co-expressing Her2/ ErbB2 antigen, fused with lamp, elicits strong antitumor effects in vivo by increasing tumor infiltration with CD8+ T cells. Cancer Research 2019;79:LB-204-0.

36 Binnewies M, Roberts EW, Kersten K, et al. Understanding the tumor immune microenvironment (time) for effective therapy. Nat Med 2018;24:541-50.

37 Drake CG, Jaffee E, Pardoll DM. Mechanisms of immune evasion by tumors. Adv Immunol 2006;90:51-81.

38 Yu P, Rowley DA, Fu Y-X, et al. The role of stroma in immune recognition and destruction of well-established solid tumors. Curr Opin Immunol 2006;18:226-31.

39 Crosby EJ, Wei J, Yang XY, et al. Complimentary mechanisms of dua checkpoint blockade expand unique T-cell repertoires and activate adaptive anti-tumor immunity in triple-negative breast tumors. Oncoimmunology 2018;7:e1421891.

40 Thakur A, Pedersen LE, Jungersen G. Immune markers and correlates of protection for vaccine induced immune responses. Vaccine 2012;30:4907-20.

41 Wimmers F, Aarntzen EHJG, Duiveman-deBoer T, et al. Longlasting multifunctional $\mathrm{CD} 8^{+} \mathrm{T}$ cell responses in end-stage melanoma patients can be induced by dendritic cell vaccination. Oncoimmunology 2016;5:e1067745.

42 Ding Z-C, Habtetsion T, Cao Y, et al. Adjuvant IL-7 potentiates adoptive $T$ cell therapy by amplifying and sustaining polyfunctional antitumor CD4+ T cells. Sci Rep 2017;7:12168.

43 Donia M, Kjeldsen JW, Andersen R, et al. PD-1+ Polyfunctional T Cells Dominate the Periphery after Tumor-Infiltrating Lymphocyte Therapy for Cancer. Clin Cancer Res 2017;23:5779-88.

44 Kyte JA, Gaudernack G, Faane A, et al. T-Helper cell receptors from long-term survivors after telomerase cancer vaccination for use in adoptive cell therapy. Oncoimmunology 2016;5:e1249090.

45 Ding Z-C, Blazar BR, Mellor AL, et al. Chemotherapy rescues tumordriven aberrant CD4+ T-cell differentiation and restores an activated polyfunctional helper phenotype. Blood 2010;115:2397-406.

46 Chikhlikar P, Barros de Arruda L, Maciel M, et al. Dna encoding an HIV-1 Gag/human lysosome-associated membrane protein-1 chimera elicits a broad cellular and humoral immune response in rhesus macaques. PLoS One 2006;1:e135.

47 Osada T, Morse MA, Hobeika A, et al. Vaccination targeting human HER3 alters the phenotype of infiltrating T cells and responses to immune checkpoint inhibition. Oncoimmunology 2017;6:e1315495.

48 Vonderheide RH, Domchek SM, Clark AS. Immunotherapy for breast cancer: what are we missing? Clin Cancer Res 2017;23:2640-6. 
49 Savas $\mathrm{P}$, Virassamy B, Ye C, et al. Single-Cell profiling of breast cancer $T$ cells reveals a tissue-resident memory subset associated with improved prognosis. Nat Med 2018;24:986-93.

$50 \mathrm{Ji} \mathrm{H}$, Chang EY, Lin KY, et al. Antigen-Specific immunotherapy for murine lung metastatic tumors expressing human papillomavirus type 16 E7 oncoprotein. Int J Cancer 1998;78:41-5.
51 Chen $\mathrm{CH}$, Wang TL, Hung CF, et al. Boosting with recombinant vaccinia increases HPV-16 E7-specific T cell precursor frequencies of HPV-16 E7-expressing DNA vaccines. Vaccine 2000;18:2015-22. 